\title{
Postischemic Lower Extremity Wounds Treated with and without Negative Pressure Dressing
}

\author{
Nazım Gümüș \\ Plastic, Reconstructive and Aesthetic Surgery Department, Medical Faculty of Cumhuriyet University, Sivas, Turkey \\ Email: gumus1970@hotmail.com
}

Received February 9, 2011; revised March 3, 2012; accepted April 25, 2012

\begin{abstract}
Background: Vacuum-assisted dressing is a noninvasive closure system of the wound, which makes localized and controlled negative pressure. Its mechanical tension reduces edema, stimulates granulation tissue formation and angiogenesis, and prepares the wound bed for closure. In this study, a patient has been presented, who suffered from serious lower extremity wounds due to arterial emboli, one of her wounds has been treated with vacuum-assisted dressing and the other with conventional dressing to evaluate the efficacy of vacuum-assisted dressing in acute ischemic wounds. Methods: A 65-year-old female patient was admitted to the emergency clinic, due to complaint of severe pain in her right lower extremity which suggested an acute arterial occlusion. She immediately underwent an embolectomy operation; however, a few days later, severe ischemia on the leg and foot became appearant. All of the necrosis was sharply debrided under sterile conditions in the operating room, and then lower leg wound was covered with vacuum-assisted dressing, also calcaneal wound was dressed with silver sulphadiazine. Results: Eighteen days after the first dressing with negative pressure, distal leg wound became ready for closure, whereas, enough granulation tissue over the calcaneal area developed merely thirty-four days later. Calcaneal wound closed spontaneously within fifty-three days. Conclusions: When dealing with this experience, using the negative pressure dressing in patient with severe lower limb wounds following arterial emboli, accelerates wound healing by means of developing the granulation tissue, and rapidly prepares the wound for closure, so it may reduce the risk of amputation.
\end{abstract}

Keywords: Negative Pressure Dressing; Ischemia; Emboli; Lower Limb; Diabetes Mellitus

\section{Introduction}

Vacuum-assisted dressing is a noninvasive closure system which generates localized and controlled negative pressure by which the mechanical tension reduces edema, stimulates granulation tissue formation and angiogenesis, and prepares the wound bed for closure by means of delayed primary or secondary intervention. The system mainly consists of a polyurethane soft sponge, a fluidimpermeable plastic film and an adjustable vacuum pump which generates a controlled continuous or intermittent subatmospheric pressure [1-3].

Vacuum-assisted closure therapy is known as a useful tool to accelerate healing of not only acute, travmatic wounds, but also non-healing chronic wounds, allowing a faster recovery with minimal hospital stay. It has also been used widely in treatment of complex wounds such as pressure ulcers, long-term dehisced wounds, venous stasis ulcers, vascular and diabetic ulcers, gunshot wounds, postoperative sternal infections, mediastinitis and defects resulting from abdominal peritoneal resections and pelvic exenterations [4-11]. In diabetic patients, although there are numerous factors leading to tissue damage and foot ulcers which are very complex and difficult to treat, successful results have been obtained with the negative pressure dressing in many studies, suggesting that it can limit the progression of tissue necrosis with meticulous wound care and essential surgical procedures and may salvage the extremity from amputation $[12,13]$. However; severe lower limb wounds in patients with vascular disease and diabetes are usually associated with poor healing rates, leading to different levels of extremity amputations, and sometimes death.

Presented here is a diabetic patient who had been suffering from multiple acute ischemic lower extremity wounds, some of which has been treated with vacuum-assisted dressing and the others with conventional dressings to evaluate the efficacy of vacuum-assisted closure in acute ischemic wounds derived from vascular obliteration.

\section{Case Report}

A 65-year-old female patient who had been suffering from deep and extensive necrosis in her right lower ex- 
tremity, was referred to our clinic from vascular surgery department. She sustained diabetes mellitus for 15 years without having any complaints about her foot, and was ambulatory before the wounds developed. As she had coronary artery disease, she underwent a coroner by-pass surgery one year ago. When she was admitted to the emergency clinic, she was complaining from severe pain in her right lower extremity which was suggesting an acute arterial occlusion, after the evaluation of the clinical and radiologic findings, she immediately underwent an embolectomy operation in which the clot was removed and then arterial blood flow of the extremity obtained. However; a few days later, severe ischemia on the leg and foot was constituted, resulting in deep and extensive tissue necrosis involving lower leg, heel and around the ankle (Figure 1). Colour doppler imaging and lower extremity arteriography clearly revealed a reduced arterial blood flow below the knee. However; a second revascularization or embolectomy procedure was not performed because of the risk of the re-occlusion of the vascular lumen with the movement of extensive and hard atherosclerotic vascular plaques. Thrombolytic treatment including systemic heparinisation was applied. An extensive and deep debridement of necrosis performed under sterile conditions in the operating room, which exposed tendon of the tibialis anterior muscle, $2 \times 1.5 \mathrm{~cm}$ surface of calcaneal bone and joint of the ankle. Lower leg wound and exposed tendon were directly covered with vacuum-assisted dressing which was applied at $125 \mathrm{~mm}$ $\mathrm{Hg}$ negative pressure, also calcaneal wound was dressed with silver sulphadiazine (Figures $\mathbf{2}$ and $\mathbf{3}$ ). Negative pressure dressing was changed every 72 hours and if necessary, extensive debridement was performed meticulously until granulation tissue became apperrant to allow wound

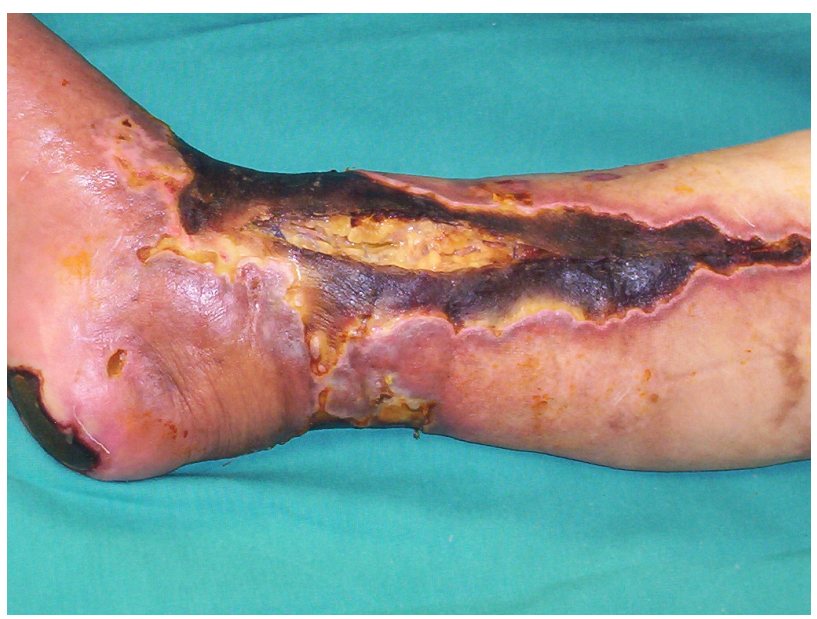

Figure 1. Appearance of the deep and extensive necrosis of the lower extremity involving lower leg, calcaneal area and around the ankle a few days after the embolectomy operation.

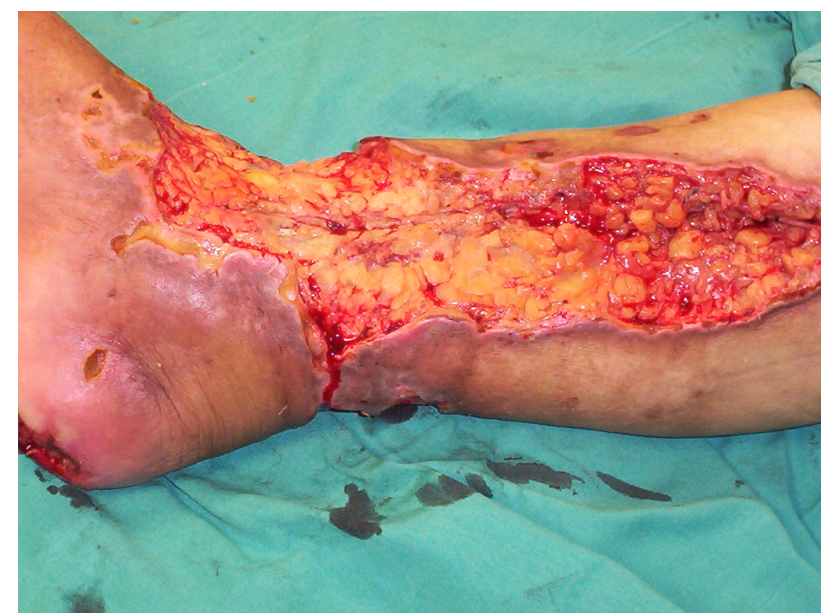

Figure 2. All of the necrosis was extensively debrided, which exposed the tendon, calcaneal bone and joint of the ankle.

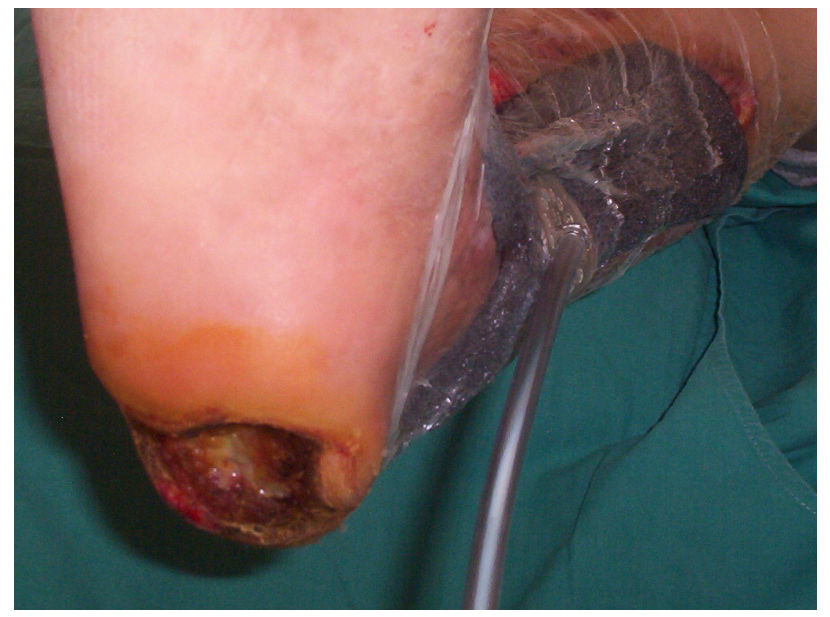

Figure 3. VAC dressing was placed just after the first extensive debridement of lower leg wound. Note that calcaneal necrosis was removed completely, leaving the calcaneal bone exposed.

closure by means of skin grafting. After the sixth dressing with negative pressure in 18 days, sufficient granulation tissue formed, permitting the coverage of wound with skin graft. Calcaneal wound was followed with daily application of silver sulphadiazine cream and when necessary, extensive excision of necrotic tissues was made every 72 hours. While negative pressure dressing was in place, daily examination of closed wound was done for any signs of infection or any other local complications.

Eighteen days after the first dressing with negative pressure, distal leg wound was ready for closure with skin grafting, so leg and ankle wounds were covered completely using split-thickness mesh skin graft, whereas at this time calcaneal wound had significant necrosis, needing serial debridements and repeated dressings (Figure 4). Thirty-four days after the beginning of the dressing with silver sulphadiazine cream, granulation tissue 
developed over the calcaneal area without remaining any necrosis, seeming suitable for skin grafting. However; heel wound closed on itself within fifty-three days (Figures 5-8). Twelve days after skin grafting of leg wound, she began walking with a heel off-loading shoe.

During the treatment, for improving her general condition, medical treatment including insulin injections, fluid replacement, intravenous antibiotics, and analgesics, was applied. Along 9 months follow-up no severe complication was observed. However, on a small area of the ankle, skin graft failed, requiring re-grafting (Figure 5). Three months after the closure of the heel wound, a small wound which was $0.5 \times 0.5 \mathrm{~cm}$ in size, developed over the calcaneal area, needing daily dressing and then it also closed spontaneously without surgical intervention.

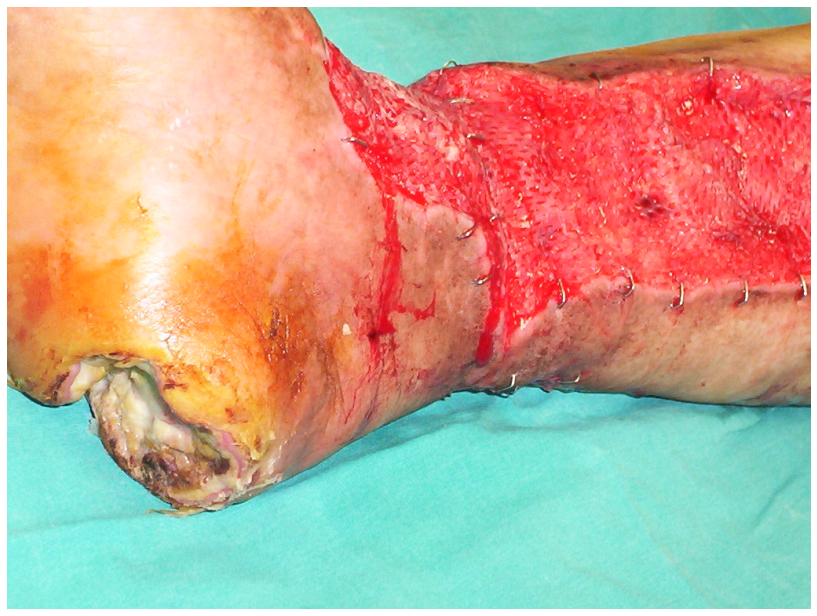

Figure 4. Intraoperative view of the extremity eighteen days after the beginning of the negative pressure dressing, while the leg and ankle wounds were covered completely with thin-thickness mesh skin graft. Note that calcaneal wound had significant necrosis, needing serial debridements and repeated dressings.

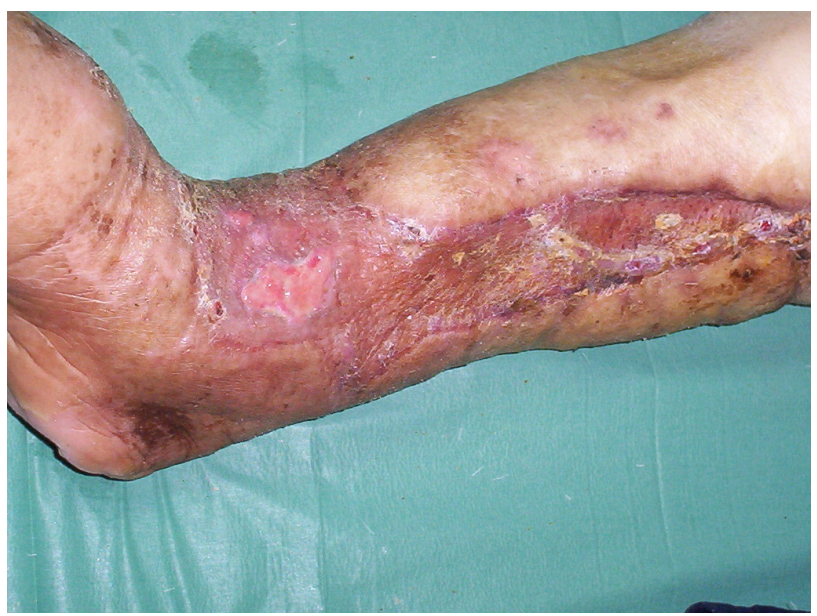

Figure 5. Appearance of the lower leg at about one month after grafting. On the small area of the ankle, skin graft failed.

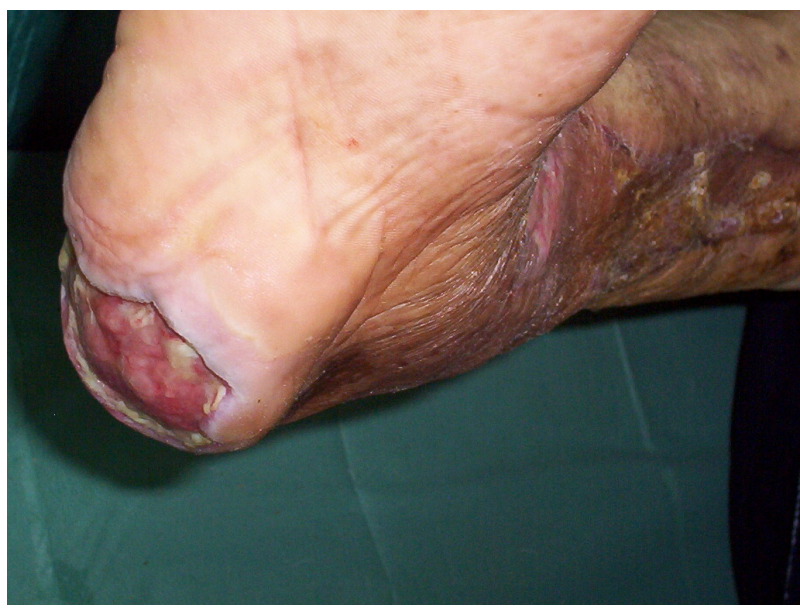

Figure 6. At the same time, view of the the calcaneal area on which enough granulation tissue developed without remaining any necrosis, seeming suitable for definitive closure.

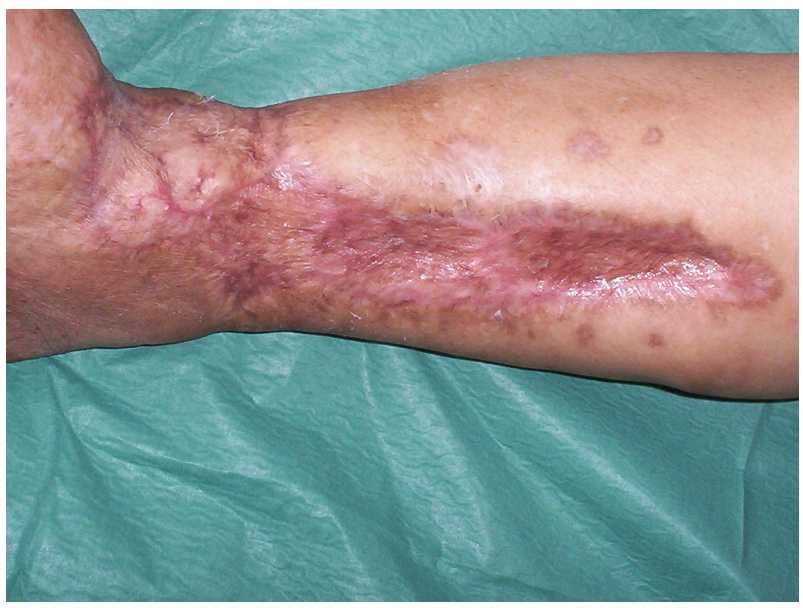

Figure 7. View of the leg seven months after skin grafting.

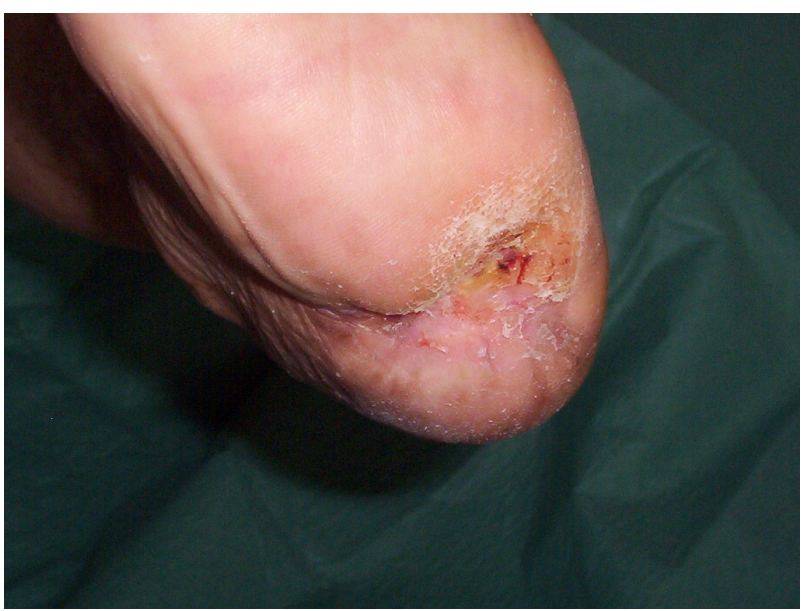

Figure 8. Appearance of the heel seven months later.

\section{Discussion}

Vacuum-assisted closure has provided an important treatment option in the management of many challenging 
wounds including pressure ulcers, long-term dehisced wounds, venous stasis ulcers, vascular and diabetic ulcers, since it was firstly utilized for chronic wounds as an alternative to conventional dressing. However, it has evolved rapidly into the treatment of acute wounds such as degloving injuries, war wounds, gunshot wounds, necrotising fasciitis, fasciotomy defects, postoperative sternal infections, contaminated wounds, and burns. Now, it is accepted as a useful tool to accelerate acute wound healing [4-11].

It has considerably changed the clinical approach to wound healing in diabetic foot ulcers, significantly reducing extremity amputations. In the treatment, foot wounds should be initially debrided for necrotic tissue completely and sufficient arterial inflow to the extremity should be accomplished with appropriate vascular procedures. Additionally, systemic antibiotics has to be given appropriately and meticulous wound care should be performed until a clean granulating bed becomes clearly appearant, which allows to cover the wounds sometimes involving bone and tendons, with skin grafts or flaps. Recently, Blume A. P. et al. presented a multicenter randomized controlled trial which enrolled 342 diabetic patients with foot ulcers to evaluate safety and clinical efficacy of negative pressure wound therapy compared with advanced moist wound therapy. In the result of the study, it has been emphasized that a significantly greater number of patients achieved complete ulcer closure and granulation tissue formation with the vacuum-assisted closure, and significant difference was not observed in ulcer-related complications such as infection, cellulitis, and osteomyelitis. Patients treated with conventional dressing had more than twice as many secondary amputations as those receiving negative pressure dressing [12]. Although our presented patient suffered from diabetes mellitus for 15 years, she had not any foot ulcers, whose complaints about lower extremity were mainly due to acute arterial occlusion leading to serious ischemic wounds exposing the tendon, bone and ankle joint. Apart from diabetes mellitus, she had an ischemic lower extremity and a large wound involving lower leg, ankle and heel, suggesting more serious conditions which threatened the lower limb viability. However; lower leg wound healed rapidly with negative pressure dressing.

Clare M. P., et al. reported their experiences with the vacuum assisted closure in 17 patients with non-healing wounds of the foot, ankle, and lower limb. Thirteen of them had diabetes mellitus, while eight had severe peripheral vascular disease. With the negative pressure dressing, wound care of the lower extremity in such wounds provided successful outcomes and revealed an acceptable alternative to the conventional dresssing [13]. Although our patient had ischemic wounds due to arterial emboli, similarly successful results were obtained with the negative pressure.

Recently, Nordmyr J., et al. presented an investigation which included one hundred and twenty one wounds treated with vacuum assisted closure therapy in patients with lower limb arterial disease. Although critical lower limb ischemia was found in $87 \%$ of the patients, complete wound healing was achieved in $66 \%$ of the wounds. It is emphasised that vacuum-assisted therapy of complex wounds in the lower limbs in patients with vascular disease is associated with high healing rates [14]. In wound healing, adequate vascular supply is the most important component, so in the absence of revascularization or sufficient blood flow, treatment of dysvascular wounds will tend to fail in every type of wound care procedure as well as negative pressure dressing. In our patient, both embolectomy and trombolytic treatment provided sufficient blood supply to the lower extremity so that either wounds could heal completely. Satisfactory and rapid healing was achieved in the wound treated with negative pressure dressing.

Although there are a few studies on using negative pressure dressing in patients with lower limb wounds due to peripheral vascular diseases in the literature, any experience is not present about the wounds following acute arterial occlusion, namely arterial emboli. This may be the first presentation according to our knowledge. Moreover; corresponding studies between two or more wounds placed near anatomical locations in the same patient, which focus on the effect of negative pressure dressing on healing comparing with conventional dressings is not found. Based on the depths of the wounds and their locations, our presented wounds cannot be considered just identical, so the healing rates for each may be different a certain degree. Moreover, dysvascularity of the posterior heel area even without any ischemic events is also well known, resulting in challenges during wound healing. However, we believe that these can show effectivity and efficacy of the technique in a corresponding manner in the extremity having two wounds treated with both dressing modalities. When compared with calcaneal wound treated with conventional dressing, the time of healing with negative pressure dressing was at about one month earlier, which was significantly important in the protection of the extremity from infections and ongoing tissue necrosis being capable of resulting in serious damage to the extremity leading to either partial or complete amputation.

When dealing with this experience, using the negative pressure dressing in patient with severe lower limb wound due to arterial emboli accelerates wound healing by means of developing the granulation tissue, reduces the risk of amputation and prepares the wound rapidly for the closure with secondary intervention. 


\section{Conflict of Interest Statement}

There are no conflict of interest statements. Neither of the authors have any financial interests, commercial associations, or other affiliations which may pose a conflict of interest to disclose. Furthermore, this paper was not supported by any external funding, nor were any special products, devices, or drugs used in the work presented.

\section{REFERENCES}

[1] L. C. Argenta, M. J. Morykwas, M. W. Marks, A. J. DeFranzo, J. A. Molnar and L. R. David, "Vacuum-Assisted Closure: State of Clinic Art," Plastic and Reconstructive Surgery, Vol. 117, No. 7, 2006, pp. 127S-142S. doi:10.1097/01.prs.0000222551.10793.51

[2] A. Braakenburg, M. C. Obdeijn, R. Feitz, I. A. L. M. Van Rooij, A. J. Van Griethuysen and J. H. G. Klinkenbijl, "The Clinical Efficacy and Cost Effectiveness of the Vacuum-Assisted Closure Technique in the Management of Acute and Chronic Wounds: A Randomized Controlled Trial," Plastic and Reconstructive Surgery, Vol. 118, No. 2, 2006, pp. 390-397. doi:10.1097/01.prs.0000227675.63744.af

[3] S. S. Scherer, G. Pietramaggiori, J. C. Mathews, M. J. Prsa, S. Huang and D. P. Orgill, "The Mechanism of Action of the Vacuum-Assisted Closure Device," Plastic and Reconstructive Surgery, Vol. 122, No. 3, 2008, pp. 786-797. doi:10.1097/PRS.0b013e31818237ac

[4] D. M. Schaffzin, J. M. Douglas, T. J. Stahl and L. E. Smith, "Vacuum-Assisted Closure of Complex Perineal Wounds," Diseases of the Colon \& Rectum, Vol. 47, No. 10, 2004, pp. 1745-1748. doi:10.1007/s10350-004-0633-9

[5] S. Cresti, M. Ouaïssi, I. Sielezneff, J. B. Chaix, N. Pirro, B. Berthet, B. Consentino and B. Sastre, "Advantage of Vacuum Assisted Closure on Healing of Wound Associated with Omentoplasty after Abdominoperineal Excision: A Case Report," World Journal of Surgical Oncology, Vol. 6, 2008, p. 136. doi:10.1186/1477-7819-6-136

[6] S. Kumar, M. E. O'Donnell, K. Khan, G. Dunne, P. D. Carey and J. Lee, "Successful Treatment of Perineal Necrotising Fasciitis and Associated Pubic Bone Osteomyelitis with the Vacuum Assisted Closure System," World Journal of Surgical Oncology, Vol. 6, 2008, pp. 67-69. doi:10.1186/1477-7819-6-67

[7] R. Bronchard, C. Vaumas, S. Lasocki, K. Jabbour, A. Geffroy, N. Kermarrec and P. Montravers, "Vacuum-Assisted Closure in the Treatment of Perineal Necrotizing Skin and Soft Tissue Infections," Intensive Care Medicine, Vol. 34, No. 7, 2008, pp. 1345-1347. doi:10.1007/s00134-008-1094-8

[8] L. Labler and O. Trentz, "The Use of Vacuum Assisted Closure in Soft Tissue Injuries after High Energy Pelvic Trauma," Langenbeck's Archives of Surgery, Vol. 392, No. 5, 2007, pp. 601-609. doi:10.1007/s00423-006-0090-0

[9] B. E. Leininger, T. E. Rasmussen, D. L. Smith, D. H. Jenkins and C. Coppola, "Experience with Wound VAC and Delayed Primary Closure of Contaminated Soft Tissue Injuries in Iraq," The Journal of Trauma and Acute Care Surgery, Vol. 61, No. 5, 2006, pp. 1207-1211.

[10] E. T. Powell IV, "The Role of Negative Pressure Wound Therapy with Reticulated Open Cell Foam in the Treatment of War Wounds," Journal of Orthopaedic Trauma, Vol. 22, Suppl. 10, 2008, pp. S138-S141. doi:10.1097/BOT.0b013e318188e27d

[11] D. J. Marsh, G. Abu-Sitta and H. Patel, "The Role of Vacuum-Assisted Wound Closure in Blast Injury," Plastic and Reconstructive Surgery, Vol. 119, No. 6, 2007, pp. 1978-1979. doi:10.1097/01.prs.0000259773.52889.68

[12] P. A. Blume, J. Walters, W. Payne, J. Ayala and J. Lantıs, "Comparison of Negative Pressure Wound Therapy Using Vacuum-Assisted Closure with Advanced Moist Wound Therapy in the Treatment of Diabetic Foot Ulcers," Diabetes Care, Vol. 31, No. 4, 2008, pp. 631-636.. doi: $10.2337 / \mathrm{dc} 07-2196$

[13] M. P. Clare, T. C. Fitzgibbons, S. T. McMullen, R. C. Stice, D. F. Hayes and L. Henkel, "Experience with the Vacuum Assisted Closure Negative Pressure Technique in the Treatment of Non-Healing Diabetic and Dysvascular Wounds," Foot \& Ankle International, Vol. 23, No. 10, 2002, pp. 896-901.

[14] J. Nordmyr, S. Svensson, M. Björck and S. Acosta, "Vacuum Assisted Wound Closure in Patients with Lower Extremity Arterial Disease. The Experience from Two Tertiary Referral-Centers," International Journal of Angiology, Vol. 28, No. 1, 2009, pp. 26-31. 